\title{
CHARACTERISTICS OF H- TO Be-LIKE IONS IMMERSED IN ELECTRON GAS
}

\author{
Marek Moneta* \\ Uniwersytet Łódzki, Katedra Fizyki Ciała Stałego \\ Pomorska 149, 90-236 Łódź, Poland
}

(Received February 26, 2001)

\begin{abstract}
The electronic characteristics of $\mathrm{H}$ - to Be-like ions immersed in degenerate electron gas were calculated. The ion was described within the HartreeFock-Slater formalism and the medium by the dielectric function. The ion's electrons repulsion, screening and exchange effects and, additionally, the self-binding of an electron due to the polarisation of the medium were included in the description. The results were compared with the reference corresponding to the characteristics for ions in vacuum.
\end{abstract}

PACS numbers: $34.50 . \mathrm{Bw}, 71.45 . \mathrm{Gm}$

\section{Introduction}

The interest in the problem arose when studying the stopping and straggling of slow ions in an electron gas [1]. It became obvious that after immersing in the electron gas, the ions alter their basic characteristics, like energy levels, excitation and ionisation energies, life times (when being excited) and all the related properties, when compared to the properties of ions in vacuum.

Several basic theoretical methods for determining characteristics of ions and molecules under vacuum environmental conditions were developed. The most important methods are variational Hartree-Fock [2] and density functional [3] methods, providing accurate results but sufficiently complicated to be rejected in stopping power theories. In this field a simpler, more transparent and instructive, and consequently less reliable, model is sufficient.

In the present paper ions having from $\mathrm{H}$ to Be electronic configurations under the electron gas environmental condition will be considered. We start from formulating the Hamiltonian describing the ion-electron gas system. Some attention will be paid to the self-binding potential of an atomic electron in the electron gas. Finally the total energies of various $1 s 2 s$ configurations will be calculated analytically and the characteristics of interest will be derived.

*e-mail: moneta@krysia.uni.lodz.pl 
The free electron gas (at $T=0 \mathrm{~K}$ ) is characterised by the one-electron radius $r_{s}\left(n=3 /\left(4 \pi r_{s}^{3}\right)\right.$ is the electron gas density). The ion consists of an atomic nucleus of atomic number $Z_{i}$ carrying $N_{i} \leq 4$ electrons.

Atomic units are used throughout.

\section{Calculation procedure}

Within the random phase approximation (RPA) the probability for transfer of the energy $\omega$ and the momentum $k$ from an ion to a degenerate free electron gas at $T=0 \mathrm{~K}$ is described by the equilibrium Lindhard dielectric function [5]: $\epsilon(u, z)=1+\left(\chi^{2} / z^{2}\right)\left[f_{1}(u, z)+\mathrm{i} f_{2}(u, z)\right]$, where the parameters are defined as $z=$ $k /\left(2 k_{\mathrm{F}}\right), u=\omega /\left(k v_{\mathrm{F}}\right), \chi^{2}=r_{s} /(\pi \alpha)$. The Fermi wave number is $k_{\mathrm{F}}=\alpha /\left(a_{0} r_{s}\right)$, the Fermi velocity $v_{\mathrm{F}}=v_{0} \alpha / r_{s}$ ( $v_{0}$ is the Bohr velocity) and $\alpha=(9 \pi / 4)^{1 / 3}$.

The conduction electrons of a solid screen the quasi-static electric potential of an ion due to dielectric response, providing screening which can be approximately described in terms of a screening function.

For ions binding $N_{i} \leq 4$ electrons we apply the Hartree-Fock-Slater (HFS) description. The total self-consistent Hamiltonian is given by

$$
H=\sum_{j}\left(-\frac{1}{2} \Delta_{j}+V_{\mathrm{pol}}\left(r_{j}\right)+V_{\mathrm{ne}}\left(r_{j}\right)+\frac{1}{2} \sum_{k \neq j} V_{\mathrm{ee}}\left(\left|r_{j}-r_{k}\right|\right)\right) .
$$

Here $V_{\text {ee }}$ and $V_{\text {ne }}$ are the exponentially screened interactions between electrons and between an electron and a nucleus, respectively

$$
\begin{aligned}
& V_{\mathrm{ne}}\left(r_{j}\right)=-Z_{i} r_{j}^{-1} \exp \left(-r_{j} k_{\mathrm{TF}}\right), \\
& V_{\mathrm{ee}}\left(\left|r_{j}-r_{k}\right|\right)=\left|r_{j}-r_{k}\right|^{-1} \exp \left(-\left|\boldsymbol{r}_{j}-\boldsymbol{r}_{k}\right| k_{\mathrm{TF}}\right) .
\end{aligned}
$$

The Thomas-Fermi wave number $k_{\mathrm{TF}}$ is related to the Fermi wave number as $k_{\mathrm{TF}}^{2}=4 k_{F} /\left(\pi a_{0}\right)$.

$V_{\text {pol }}$ is the self-binding potential acting on an electron from polarisation wake induced in the electron gas by this electron

$$
V_{\text {pol }}(r)=\int_{\mathcal{R}^{3}} \mathrm{~d}^{3} r^{\prime} \psi_{a}\left(r^{\prime}\right)\left|r-r^{\prime}\right|^{-1}\left[\exp \left(-\left|r-r^{\prime}\right| k_{\mathrm{TF}}\right)-1\right] \psi_{a}\left(r^{\prime}\right)
$$

Physically, this phenomenological Hamiltonian gives account of an approximate, long wavelength screening exerted by the electron gas on an ion, instead of the response provided by the full dielectric function $\epsilon(k, \omega)$.

We assume that the solutions of Eq. (1), in a form of HFS determinants, are built from the $1 s$ and $2 s$ one-electron trial functions:

$$
\begin{aligned}
& \psi_{1 s}(r)=\pi^{-1 / 2}(Z)^{3 / 2} \exp (-Z r), \\
& \psi_{2 s}(r)=\pi^{-1 / 2}(Z / 2)^{3 / 2}(1-Z r / 2) \exp (-Z r / 2) .
\end{aligned}
$$

We call $Z$ the size parameter of the electron distribution. This parameter is modified when the ion from the vacuum enters a solid. We determine $Z=Z_{\text {min }}$ from the variational condition of minimum for the expectation values of the total 
Hamiltonian $\bar{H}$. For different $1 s 2 s$ configurations of excited (Ion*) ions and ions in the ground state (Ion) we have

$$
\begin{aligned}
& \bar{H}\left(\mathrm{H} \equiv 1 s^{1}\right)=E_{1 s}+P_{1 s}, \\
& \bar{H}\left(\mathrm{H}^{*} \equiv 1 s^{0} 2 s^{1}\right)=E_{2 s}+P_{2 s}, \\
& \bar{H}\left(\mathrm{He} \equiv 1 s^{2}\right)=2 E_{1 s}+2 P_{1 s}+V_{1 s 1 s}, \\
& \bar{H}\left(\mathrm{He}^{*} \equiv 1 s^{1} 2 s^{1}\right)=E_{1 s}+P_{1 s}+E_{2 s}+P_{2 s}+V_{1 s 2 s}-A_{1 s 2 s}, \\
& \bar{H}\left(\mathrm{He}^{* *} \equiv 1 s^{0} 2 s^{2}\right)=2 E_{2 s}+2 P_{2 s}+V_{2 s 2 s}, \\
& \bar{H}\left(\mathrm{Li}^{2} \equiv 1 s^{2} 2 s^{1}\right)=2 E_{1 s}+2 P_{1 s}+E_{2 s}+P_{2 s}+V_{1 s 1 s}+2 V_{1 s 2 s}-A_{1 s 2 s}, \\
& \bar{H}\left(\mathrm{Li}^{*} \equiv 1 s^{1} 2 s^{2}\right)=E_{1 s}+P_{1 s}+2 E_{2 s}+2 P_{2 s}+V_{2 s 2 s}+2 V_{1 s 2 s}-A_{1 s 2 s}, \\
& \bar{H}\left(\mathrm{Be} \equiv 1 s^{2} 2 s^{2}\right) \\
& \quad=2 E_{1 s}+2 P_{1 s}+2 E_{2 s}+2 P_{2 s}+V_{1 s 1 s}+V_{2 s 2 s}+4 V_{1 s 2 s}-2 A_{1 s 2 s} .
\end{aligned}
$$

The one-electron energy $(E)$, the direct Coulomb $(V)$, the polarisation $(P)$ and the exchange $(A)$ integrals were calculated analytically, and are presented in Appendix and their values for an extremely dense or diluted electron gas can be obtained from Table I.

TABLE I

Values of the intergrals for $\mathrm{H}-$ to Be-like ions in vacuum and a dense electron gas.

\begin{tabular}{c|c|c}
\hline \hline Integrals & Vacuum, $r_{s}=\infty, k_{\mathrm{TF}}=0$ & Dense gas, $r_{s}=0, k_{\mathrm{TF}}=\infty$ \\
\hline$E_{1 s}$ & $Z^{2} / 2-Z Z_{i}$ & $Z^{2} / 2$ \\
$E_{2 s}$ & $Z^{2} / 8-Z Z_{i} / 4$ & $Z^{2} / 8$ \\
$V_{1 s 1 s}$ & $Z 5 / 8$ & 0 \\
$P_{1 s}$ & 0 & $-Z 5 / 8$ \\
$V_{2 s 2 s}$ & $Z 77 / 512$ & 0 \\
$P_{2 s}$ & 0 & $-Z 77 / 512$ \\
$V_{1 s 2 s}$ & $Z 68 / 324$ & 0 \\
$A_{1 s 2 s}$ & $Z 16 / 729$ & 0
\end{tabular}

It was assumed, and it was as a criterion, that for the ion in an electron gas the total energy in the minimum has to be negative, if the ion is to be stable against the decay into other, more favourable energetically, electronic configurations. 


\section{Results and discussion}

\subsection{Ion in vacuum}

When ions with various atomic numbers $Z_{i}$ having $\mathrm{H}$ - to Be-like electronic configurations are placed in the vacuum $\left(r_{s}=\infty\right)$ then the screening of an electron gas is neglected $\left(k_{\mathrm{TF}}=0\right)$, and only the screening and exchange interactions related to atomic electrons remain effective. Also, the self-binding is absent in vacuum.

The total energies $\bar{H}$ 's of Eq. (5), explicitly given in Table I, reach minima (calculated from $\partial \bar{H} / \partial Z=0$ ) at the values $Z_{\text {min }}$ presented in Table II for ions in the ground and excited states. The screening by ionic electrons and the exchange interaction are more important for an ion with a small atomic number, since $Z_{\min }$ is equal to the difference between the ion atomic number $Z_{i}$ and some specific screening number of the order of unity. The total energy is proportional to $Z_{\mathrm{min}}^{2}$ with some factor given in the last column of Table II depending on the electronic configuration.

TABLE II

The minimum energy parameter $Z_{\min }$ and the total energy $\bar{H}$ of Eq. (5) for H- to Be-like ions.

\begin{tabular}{|c|c|c|c|}
\hline X-like ions & $Z_{\min }\left(r_{s}=\infty\right)$ & $Z_{\min }\left(r_{s}=0\right)$ & $\bar{H} / Z_{\min }^{2}$ \\
\hline $\mathrm{H}\left(1 s^{1} 2 s^{0}\right)$ & $Z_{i}$ & 0.625 & $-1 / 2$ \\
\hline $\mathrm{H}^{*}\left(1 s^{0} 2 s^{1}\right)$ & $Z_{i}$ & 0.602 & $-1 / 8$ \\
\hline $\operatorname{He}\left(1 s^{2} 2 s^{0}\right)$ & $Z_{i}-0.31250$ & 0.625 & -1 \\
\hline $\mathrm{He}^{*}\left(1 s^{1} 2 s^{1}\right)$ & $Z_{i}-0.15034$ & 0.620 & $-5 / 8$ \\
\hline $\mathrm{He}^{* *}\left(1 s^{0} 2 s^{2}\right)$ & $Z_{i}-0.30078$ & 0.602 & $-1 / 4$ \\
\hline $\operatorname{Li}\left(1 s^{2} 2 s^{1}\right)$ & $Z_{i}-0.45458$ & 0.622 & $-9 / 8$ \\
\hline $\mathrm{Li}^{*}\left(1 s^{1} 2 s^{2}\right)$ & $Z_{i}-0.36546$ & 0.617 & $-3 / 4$ \\
\hline $\operatorname{Be}\left(1 s^{2} 2 s^{2}\right)$ & $Z_{i}-0.62840$ & 0.620 & $-5 / 4$ \\
\hline
\end{tabular}

For a given atomic number $Z_{i}$ the total energy depends on the electronic configuration and the number $N_{i}$ of bound electrons. This dependence is drawn in Fig. 1 for ions with $Z_{i}=1$ to $Z_{i}=4$. It turns out that there is a local minimum of the total energy for $N_{i}=Z_{i}$, i.e. removal or attachment of an electron demands some amount of energy. The total energy of an ion can be simply calculated starting from the analytical relations given in Table II. For instance, a proton as a nucleus can be analysed by taking $Z_{i}=1$, so we have for various electronic configurations: $\bar{H}\left(\mathrm{H}\left(1 s^{1} 2 s^{0}\right)\right)=-13.6 \mathrm{eV}, \bar{H}\left(\mathrm{H}\left(1 s^{2} 2 s^{0}\right)\right)=-12.9 \mathrm{eV}, \bar{H}\left(\mathrm{H}\left(1 s^{2} 2 s^{1}\right)\right)=-9.1 \mathrm{eV}$, $\bar{H}\left(\mathrm{H}\left(1 s^{2} 2 s^{2}\right)\right)=-4.7 \mathrm{eV}$, associated with adequate $Z_{\min }$ 's. This means that all these electronic configurations are stable in vacuum. In the case of Li-like ions, $Z_{i}=3$, we get $\bar{H}\left(\mathrm{Li}^{+}\left(1 s^{2} 2 s^{0}\right)\right)=-196.4 \mathrm{eV}, \bar{H}\left(\operatorname{Li}\left(1 s^{2} 2 s^{1}\right)\right)=-198.3 \mathrm{eV}$ and $\bar{H}\left(\mathrm{Li}^{-}\left(1 s^{2} 2 s^{2}\right)\right)=-191.2 \mathrm{eV}$, so the minimum is very shallow. 


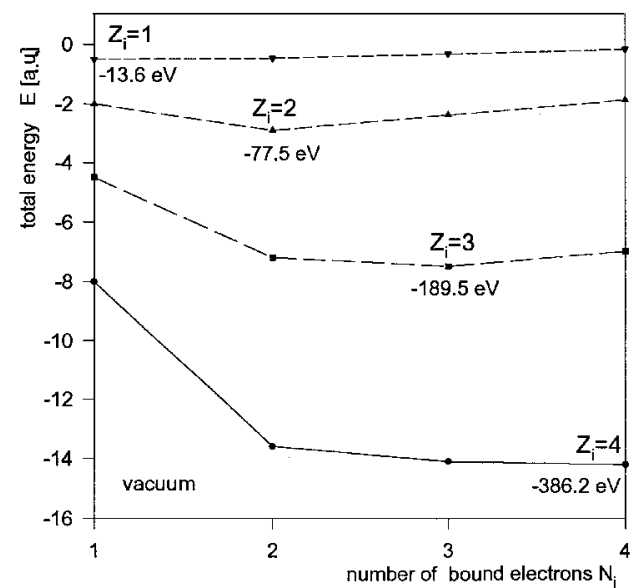

Fig. 1. The dependence of total energy of ions with atomic numbers $Z_{i}$ on the number $N_{i}$ of bound electrons for $\mathrm{H}$ - to Be-like electronic configurations.

The present results can be compared with the Hartree-Fock-Slater calculations [2] performed for atoms and both positive $\left(\mathrm{X}^{1+}\right)$ and negative $\left(\mathrm{X}^{1-}\right)$ ions in vacuum. The comparison, given in Table III, shows minima about $1-2 \%$ deeper as related to the values obtained within the present model. For ions with various electronic configurations Clementi and Roetti [2] also obtained shallow minima for $N_{i}=Z_{i}$. In the case of Li-like ions, $Z_{i}=3$, they got $\bar{H}\left(\mathrm{Li}^{+}\left(1 s^{2} 2 s^{0}\right)\right)=-196.8 \mathrm{eV}$, $\bar{H}\left(\mathrm{Li}\left(1 s^{2} 2 s^{1}\right)\right)=-202.2 \mathrm{eV}$ and $\bar{H}\left(\mathrm{Li}^{-}\left(1 s^{2} 2 s^{2}\right)\right)=-202.0 \mathrm{eV}$. The calculated total energy for He atom, $\bar{H}\left(\mathrm{He}\left(1 s^{2} 2 s^{0}\right)\right)=-77.45 \mathrm{eV}$, is slightly above the reference value of $-77.83 \mathrm{eV}[2]$.

TABLE III

The total energy of $\mathrm{H}$ to Be atoms (hartree). No self-binding.

\begin{tabular}{l|c|c|c|c|c|c}
\hline \hline Atoms & $r_{s}=1.0$ & $r_{s}=2.0$ & $r_{s}=3.0$ & $r_{s}=5.0$ & $r_{s}=\infty$ & Ref. [2] \\
\hline $\mathrm{H}\left(1 s^{1} 2 s^{0}\right)$ & + & + & -0.0155 & -0.0678 & -0.5000 & \\
$\mathrm{H}^{*}\left(1 s^{0} 2 s^{1}\right)$ & + & + & + & + & -0.1234 & \\
$\mathrm{He}\left(1 s^{2} 2 s^{0}\right)$ & -0.1604 & -0.6072 & -0.8837 & -1.2146 & -2.8430 & -2.8617 \\
$\mathrm{He}^{*}\left(1 s^{1} 2 s^{1}\right)$ & -0.0745 & -0.3562 & -0.5409 & -0.7720 & -2.1336 & \\
$\mathrm{He}^{* *}\left(1 s^{0} 2 s^{2}\right)$ & + & + & + & -0.0102 & -0.7172 & \\
$\mathrm{Li}\left(1 s^{2} 2 s^{1}\right)$ & -1.5266 & -2.6659 & -3.2874 & -3.9948 & -7.2797 & -7.4327 \\
$\mathrm{Li}^{*}\left(1 s^{1} 2 s^{2}\right)$ & -0.6916 & -1.3934 & -1.8123 & -2.3226 & -5.1962 & \\
$\operatorname{Be}\left(1 s^{2} 2 s^{2}\right)$ & -4.2982 & -6.3349 & -7.4259 & -8.6336 & -14.1940 & -14.5730
\end{tabular}

In vacuum the energy required for ion excitation or for electron detachment can be calculated as a difference of adequate $\bar{H}$ 's, and is given in the last but one column of Table IV and compared with the available reference values [2] given in the last column of the table. For example the excitation $\mathrm{He}\left(1 s^{2} 2 s^{0}\right) \rightarrow \mathrm{He}^{*}\left(1 s^{1} 2 s^{1}\right)$ 
demands $19.29 \mathrm{eV}$, and electronic detachment $\mathrm{He}\left(1 s^{2} 2 s^{0}\right) \rightarrow \mathrm{He}^{+}\left(1 s^{1} 2 s^{0}\right)$ demands $23.06 \mathrm{eV}$, whereas the recommended He ionization energy obtained from photoelectron measurements is $24.58 \mathrm{eV}$ [4].

TABLE IV

The selected excitation and ionisation energies of $\mathrm{H}$ to $\mathrm{Be}$ atoms (hartree). No self-binding.

\begin{tabular}{l|r|r|r|r|r}
\hline \hline \multicolumn{1}{c|}{ Reaction } & $r_{s}=1.0$ & $r_{s}=2.0$ & $r_{s}=5.0$ & $r_{s}=\infty$ & Ref. [2] \\
\hline $\mathrm{H}\left(1 s^{1} 2 s^{0}\right) \rightarrow \mathrm{H}^{+}\left(1 s^{0} 2 s^{0}\right)$ & & & 0.067797 & 0.49844 & \\
$\mathrm{He}\left(1 s^{2} 2 s^{0}\right) \rightarrow \mathrm{He}^{+}\left(1 s^{1} 2 s^{0}\right)$ & -0.0129 & 0.1159 & 0.3082 & 0.8461 & 0.8617 \\
$\mathrm{He}\left(1 s^{2} 2 s^{0}\right) \rightarrow \mathrm{He}^{2+}\left(1 s^{0} 2 s^{0}\right)$ & 0.1604 & 0.6072 & 1.2145 & 2.8430 & 2.8617 \\
$\mathrm{He}\left(1 s^{2} 2 s^{0}\right) \rightarrow \mathrm{He}^{*}\left(1 s^{1} 2 s^{1}\right)$ & 0.0859 & 0.0663 & 0.4425 & 0.7094 & \\
$\mathrm{He}\left(1 s^{2} 2 s^{0}\right) \rightarrow \mathrm{He}^{* *}\left(1 s^{0} 2 s^{2}\right)$ & 0.1604 & 0.6072 & 1.2044 & 2.1258 & \\
$\mathrm{Li}\left(1 s^{2} 2 s^{1}\right) \rightarrow \mathrm{Li}^{+}\left(1 s^{2} 2 s^{0}\right)$ & 1.5266 & 2.6659 & 3.9605 & 6.8086 & \\
$\mathrm{Li}\left(1 s^{2} 2 s^{1}\right) \rightarrow \mathrm{Li}^{*+}\left(1 s^{1} 2 s^{1}\right)$ & 0.5941 & 1.0036 & 1.4336 & 2.2122 & \\
$\mathrm{Li}\left(1 s^{2} 2 s^{1}\right) \rightarrow \mathrm{Li}^{2+}\left(1 s^{1} 2 s^{0}\right)$ & 0.2955 & 0.3548 & 1.2708 & 2.7844 & 2.9327 \\
$\mathrm{Li}\left(1 s^{2} 2 s^{1}\right) \rightarrow \mathrm{Li}^{3+}\left(1 s^{0} 2 s^{0}\right)$ & 1.5269 & 2.5659 & 3.9257 & 7.2797 & 7.4327 \\
$\mathrm{Be}\left(1 s^{2} 2 s^{2}\right) \rightarrow \mathrm{Be}^{+}\left(1 s^{2} 2 s^{1}\right)$ & -0.4785 & -0.4603 & -0.3729 & 0.0668 & 0.2956 \\
$\mathrm{Be}\left(1 s^{2} 2 s^{2}\right) \rightarrow \mathrm{Be}^{*+}\left(1 s^{1} 2 s^{2}\right)$ & 1.9742 & 2.6816 & 3.3350 & 4.3006 & \\
$\mathrm{Be}\left(1 s^{2} 2 s^{2}\right) \rightarrow \mathrm{Be}^{2+}\left(1 s^{2} 2 s^{0}\right)$ & -1.0153 & -1.8994 & -0.6578 & 0.6073 & \\
$\mathrm{Be}\left(1 s^{2} 2 s^{2}\right) \rightarrow \mathrm{Be}^{* * 2+}\left(1 s^{0} 2 s^{2}\right)$ & 4.2771 & 6.2227 & 8.1202 & 10.7836 & \\
$\mathrm{Be}\left(1 s^{2} 2 s^{2}\right) \rightarrow \mathrm{Be}^{* 2+}\left(1 s^{1} 2 s^{1}\right)$ & 1.5379 & 2.2997 & 3.1256 & 4.9425 & \\
$\mathrm{Be}\left(1 s^{2} 2 s^{2}\right) \rightarrow \mathrm{Be}^{3+}\left(1 s^{1} 2 s^{0}\right)$ & 1.0550 & 1.4075 & 3.0991 & 6.2002 & 6.5730 \\
$\mathrm{Be}\left(1 s^{2} 2 s^{2}\right) \rightarrow \mathrm{Be}^{* 3+}\left(1 s^{0} 2 s^{1}\right)$ & 4.2866 & 6.1958 & 8.3297 & 12.2002 & \\
$\mathrm{Be}\left(1 s^{2} 2 s^{2}\right) \rightarrow \mathrm{Be}^{4+}\left(1 s^{0} 2 s^{0}\right)$ & 4.2982 & 6.3349 & 8.6336 & 14.1940 & 14.5730 \\
& & & &
\end{tabular}

There is a quite correct relation between the results of the present simple model of the total energy calculation for an ion in vacuum and the reference values [2] based on different basis sets (full HFS, double and single zeta functions). The agreement would certainly have been better, if we took, for instance, two variational parameters, (say $Z_{1}$, and $Z_{2}$ ) instead of single $Z$, in the wave functions of Eq. (4).

\subsection{Ion in electron gas}

The point of interest of the present paper is to analyse how the spectroscopic characteristics of atoms are modified, when the object is immersed in a uniform electron gas at $T=0 \mathrm{~K}$, characterised by a density parameter $r_{s}$.

As previously, it is assumed that an ion exists as an integral object as long, as its total energy in the minimum remains negative. This condition is fulfilled over the whole $r_{s}$ range, provided the self-binding given by Eq. (3) is incorporated in the model. If the self-binding is suspended, the total energy acquires zero 
at some $r_{s}$, causing the ion to disintegrate, as the energy gets positive values when $r_{s} \rightarrow 0$.

In an electron gas, within a description taking into account the self-binding interaction or not, the $Z_{\text {min }}$ parameter was determined by calculating a numerical minimum of the appropriate $\bar{H}$ from Eq. (5). It turns out that $Z_{\text {min }}$ depends on $Z_{i}, N_{i}$ and, additionally, on $r_{s}$. In order to illustrate this behaviour, the $Z_{\min }$, as a function of $r_{s}$ and $Z_{i}$, is drawn in Fig. 2 for Li-like ions remaining in the
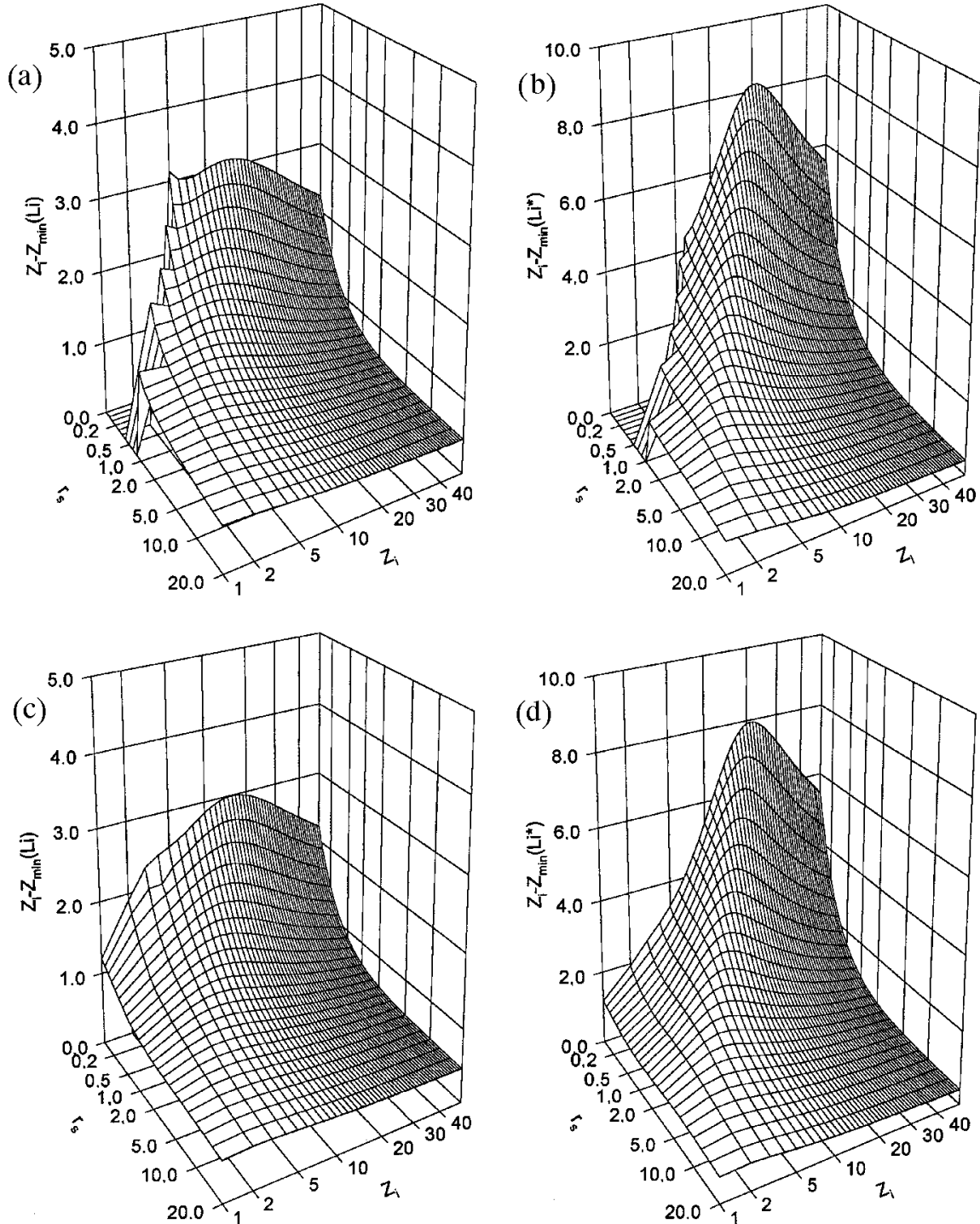

Fig. 2. $Z_{\min }$ as a function of $r_{s}$ and $Z_{i}$ for Li-like ions. Figures (a) and (b) without, and figures $(c)$ and $(d)$ with the self-binding interaction. 
ground (a) and in the excited (b) states without self-binding and the same with self-binding (c), (d).

By analysing Fig. 2 it is seen that as $r_{s}$ increases (dilute gas) for a given $Z_{i}$, $Z_{\min }$ also increases to the atomic screening numbers given in the second column of Table II for $r_{s}=\infty$. Due to an increase in $Z_{\min }$, the atomic electron trial functions of Eq. (4) tend to be more localised around the nucleus, as in the case of an ion in vacuum. A decrease in $r_{s}$ (dense gas) causes a restricted spreading of the functions in the electron gas.

While comparing drawings describing different states of ion excitation, it should be noted that $Z_{\text {min }}$, calculated for the same $\left(r_{s}, Z_{i}\right)$ parameters, is much smaller for the excited ions than for the ions in the ground state: $Z_{\min }(\mathrm{He})>$ $Z_{\min }\left(\mathrm{He}^{*}\right)>Z_{\min }\left(\mathrm{He}^{* *}\right)$. It simply means that the excited ions spread more in space than the ions in the ground state.

$Z_{\text {min }}$ is used in Eq. (5) to calculate $\bar{H}\left(Z_{\min }\right)$. In a dilute and dense electron gas $\bar{H}_{\min }$ is directly proportional to $Z_{\min }^{2}$. As an example, the dependence of $\bar{H}_{\min } / Z_{\min }^{2}$ upon $Z_{i}$ and $r_{s}$ for $\operatorname{Li}\left(1 s^{2} 2 s^{1}\right)$ atom in the ground state and for $\mathrm{Li}^{*}\left(1 s^{1} 2 s^{2}\right)$ excited atom are drawn in Fig. 3, separating the cases without $(\mathrm{a}, \mathrm{b})$ and with $(c, d)$ binding of electrons to polarisation wakes. First of all, we note that this function is not constant, equal to the value in vacuum. As $r_{s}$ decreases, for a given $Z_{i}, Z_{\min }$ also decreases causing an increase of the functions in the figures.

From the analysis of $\bar{H}_{\min } / Z_{\min }^{2}$ upon $Z_{i}$ and $r_{s}$, we found that due to accounting for the self-binding of each electron to the polarisation wake, all the ions in the electron gas remain stable against the decay over the whole $r_{s}$ and $Z_{i}$ range considered, contrary to the experiment. For a very dense electron gas $\left(r_{s}=0\right) Z_{\text {min }}$ is independent of $Z_{i}$, as given in Table II. For example, for H-like ions a minimum of energy is $-5.3 \mathrm{eV}$, and for Be-like ions is $-13.1 \mathrm{eV}$, and these values decrease to the values appropriate for these ions in vacuum, as $r_{s} \rightarrow \infty$. By setting $Z_{i}=0$, we can calculate electron self-binding energies, the values which can be associated with appropriate work functions, ranging for real metals from $-4 \mathrm{eV}$ to $-5 \mathrm{eV}$.

On the other hand, in the description of the ion the suspending of the self-binding causes an increase in the ion energy for the dense electron gas, reaching zero at some $r_{s}$ (as $r_{s} \rightarrow 0$ ) and fulfilling the criterion for the ion decay. In this case, for instance, in agreement with expectation from the experiment, $\mathrm{H}$ atom cannot exist in the electron gas below $r_{s}=2$ and $\mathrm{H}^{*}$ below $r_{s}=10$, approximately. The selected results of calculations within the present model (excluding self-binding) regarding the minimum total energy of Eq. (5) for $\mathrm{H}$ to Be atoms in dependence of the electron gas density parameter $r_{s}$ are presented in Table III and compared with the available reference values [2]. Generally, the total energy of an atom increases along with an increase in the electron gas density. Among the atoms investigated, only $\mathrm{He}, \mathrm{He}^{*}, \mathrm{Li}_{2} \mathrm{Li}^{*}$, and $\mathrm{Be}$ can, in principle, exist and be stable in an electron gas, whose density corresponds to the density of real metals, i.e. from $r_{s}=1$ to $r_{s}=3$. The total energy of $\mathrm{H}, \mathrm{H}^{*}$, and $\mathrm{He}^{* *}$ is positive in this density region causing the atoms to decay spontaneously, for instance by emitting electrons. The results on energies required for excitation or ionisation of $\mathrm{H}$ to $\mathrm{Be}$ atoms are enclosed in Table IV and related to the reference data [2]. The plus sign means that the energy has to be provided in order to carry out the transition, 

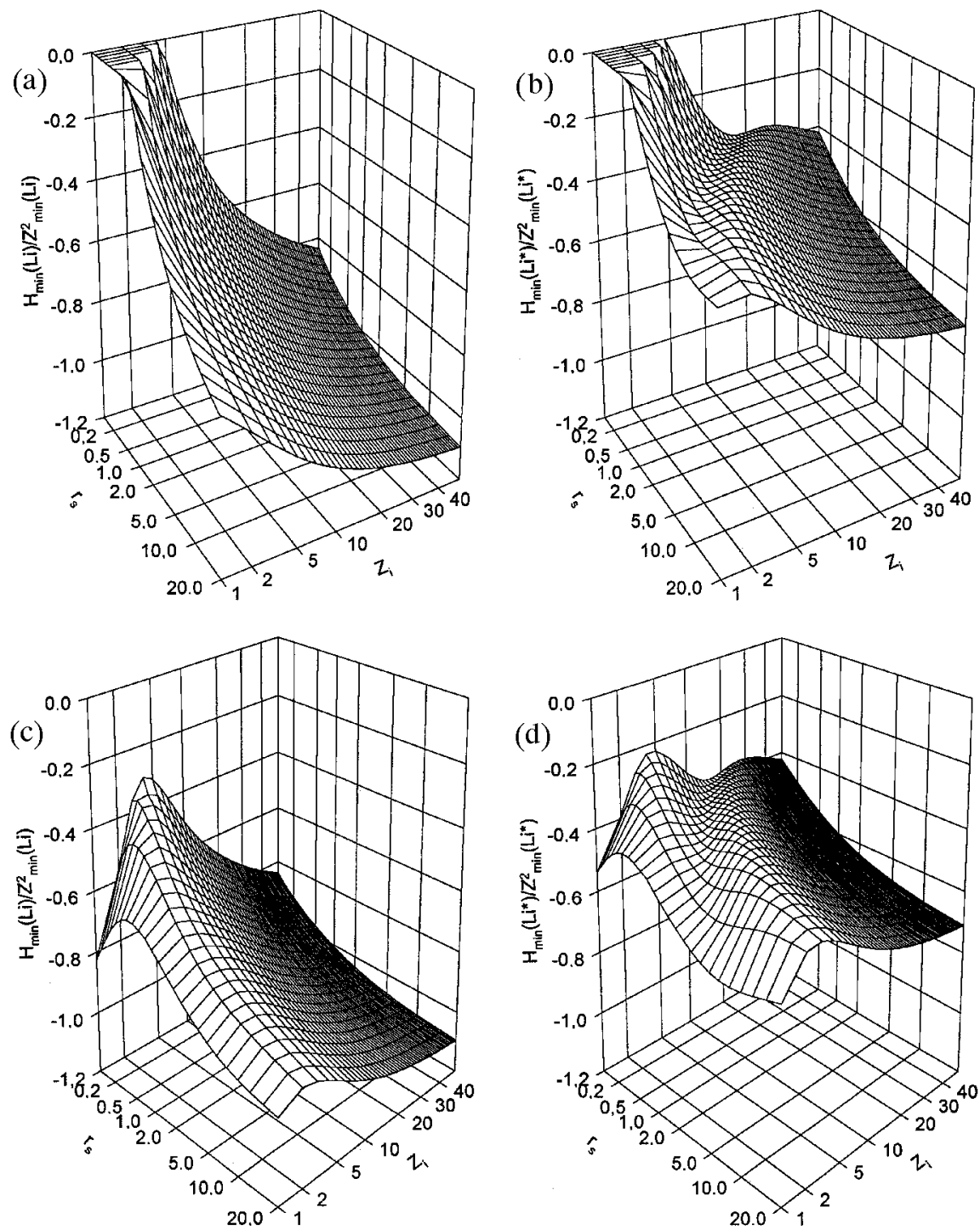

Fig. 3. $H_{\min } / Z_{\min }^{2}$ as a function of $r_{s}$ and $Z_{i}$ for Li-like ions. Figures (a) and (b) without, and figures (c) and (d) with the self-binding interaction.

whereas the minus sign means that the energy is gained in the spontaneous transition. For example, it is seen from Table III that for total collisional stripping off the He atom, prepared initially in the ground state, the energy required is strongly dependent on the interaction with the medium in which the atom is immersed; in vacuum the energy of $77.3 \mathrm{eV}$ is necessary, whereas in the electron gas of $r_{s}=2$ the energy needed is about $16.5 \mathrm{eV}$. For a complete stripping off the Be atom the corresponding values are $172.3 \mathrm{eV}$ and $380 \mathrm{eV}$, respectively. 


\section{Conclusions}

The spectroscopic properties of $\mathrm{H}$ - to Be-like ions immersed in an electron gas were analysed, and shown to depend on the ion atomic number, the number of bound electrons, and the configuration they create, and on the density of the electron gas. It was shown that the denser electron gas, the higher energy of the ion in the minimum found from the variational principle. Also, the energies required for ionisation or excitation of an ion kept in an electron gas decrease, as the electron gas density increases. An answer to the questions whether the electronic configuration of the ion remains stable in the electron gas up to an extremely high density, or whether there is a critical density above which the ion total energy becomes positive and the ion disintegrates, depends on whether binding of an electron to the wake potential which this electron induces in the electron gas is included in the description or this self-binding is suspended. A form of the potential is imposed by Eq. (3) which defines this interaction as a difference of potentials produced by an electron in the electron gas and in vacuum. The electron is represented by the trial function, and the electron gas is described by a dielectric function generating static screening approximated in this model by exponential screening. Despite of the fact that the present model equipped with this self-binding interaction can produce a quantity which may be interpreted as a work function, the resultant stability of atoms immersed in the dense electron gas is in clear disagreement with experiment. This makes us suspend this component of the model. On the other hand, there is an evaluation of a number of bound states $n^{\prime}$ in a screened Coulomb potential, corresponding to the screening exerted by the electron gas, performed numerically by Rogers et al. [8]. They found $n^{\prime}=0.5829+0.4993 Z_{i} / k_{\mathrm{TF}} \cdot n^{\prime}=1.36$ for $Z_{i}=1$ and $r_{s}=1$ which suggests that the hydrogen atom should be stable in a real metal.

\section{Appendix}

The following one-electron energy $(E)$, the Coulomb $(V)$, the polarisation $(P)$, and the exchange $(A)$ integrals:

$$
\begin{aligned}
& E_{a}=\int_{\mathcal{R}^{3}} \mathrm{~d}^{3} r \psi_{a}(r)\left(-\frac{1}{2} \Delta-\frac{Z_{i}}{r} \exp \left(-r k_{\mathrm{TF}}\right)\right) \psi_{a}(r), \\
& V_{a b}=\int_{\mathcal{R}^{3}} \mathrm{~d}^{3} r \int_{\mathcal{R}^{3}} \mathrm{~d} r^{\prime}\left|\psi_{a}(r)\right|^{2}\left|r-r^{\prime}\right|^{-1} \exp \left(-\left|r-r^{\prime}\right| k_{\mathrm{TF}}\right)\left|\psi_{b}\left(r^{\prime}\right)\right|^{2}, \\
& P_{a}=\int_{\mathcal{R}^{3}} \mathrm{~d}^{3} r \int_{\mathcal{R}^{3}} \mathrm{~d}^{3} r^{\prime}\left|\psi_{a}(r)\right|^{2}\left|r-r^{\prime}\right|^{-1}\left[\exp \left(-\left|r-r^{\prime}\right| k_{\mathrm{TF}}\right)-1\right]\left|\psi_{a}\left(r^{\prime}\right)\right|^{2}, \\
& A_{a b}=\int_{\mathcal{R}^{3}} \mathrm{~d}^{3} r \int_{\mathcal{R}^{3}} \mathrm{~d}^{3} r^{\prime} \psi_{a}^{*}(r) \psi_{b}^{*}\left(r^{\prime}\right)\left|r-r^{\prime}\right|^{-1} \exp \left(-\left|r-r^{\prime}\right| k_{\mathrm{TF}}\right) \psi_{a}\left(r^{\prime}\right) \psi_{b}(r),
\end{aligned}
$$

with the functions 


$$
\begin{aligned}
& \psi_{1 s}(r)=\pi^{-1 / 2}(Z)^{3 / 2} \exp (-Z r), \\
& \psi_{2 s}(r)=\pi^{-1 / 2}(Z / 2)^{3 / 2}(1-Z r / 2) \exp (-Z r / 2),
\end{aligned}
$$

were calculated analytically as functions of $Z\left(\gamma=k_{\mathrm{TF}} / Z\right.$ and $\left.\gamma^{\prime}=2 \gamma / 3\right)$

$$
\begin{aligned}
& E_{1 s}=\frac{Z^{2}}{2}-\frac{Z_{i} Z}{(1+\gamma / 2)^{2}}, \\
& E_{2 s}=\frac{Z^{2}}{8}-\frac{Z_{i} Z}{4(1+\gamma)^{2}}\left(2-\frac{4}{(1+\gamma)}+\frac{3}{(1+\gamma)^{2}}\right) \\
& V_{1 s 1 s}=Z \frac{5+2 \gamma+\gamma^{2} / 4}{8(1+\gamma / 2)^{4}}, \\
& P_{1 s}=V_{1 s 1 s}-5 Z / 8, \\
& V_{2 s 2 s}=Z \frac{77+104 \gamma+227 \gamma^{2}+144 \gamma^{3}+123 \gamma^{4}+40 \gamma^{5}+5 \gamma^{6}}{512(1+\gamma)^{8}}, \\
& P_{2 s}=V_{2 s 2 s}-77 Z / 512, \\
& V_{1 s 2 s}=Z \frac{4}{81} \frac{17+4 \gamma+21 \gamma^{2}+8 \gamma^{3}+\gamma^{4}}{(1+\gamma)^{4}(2+\gamma)^{2}}, \\
& A_{1 s 2 s}=Z \frac{16}{729} \frac{3-25 \gamma^{\prime 2}+150 \gamma^{\prime 4}-256 \gamma^{\prime 5}+150 \gamma^{\prime 6}-25 \gamma^{\prime 8}+3 \gamma^{\prime 10}}{3\left(1-\gamma^{\prime 2}\right)^{6}}
\end{aligned}
$$

in hartree $\left(e^{2} / a_{0}\right)$ units. For $\gamma^{\prime}=1$ the exchange integral $A$ assumes the finite value $A_{1 s 2 s}=Z(16 / 729)(5 / 12)$.

\section{References}

[1] M. Moneta, Acta Phys. Pol. A 93, 775 (1998).

[2] E. Clementi, C. Roetti, Atomic Data and Nuclear Data Tables 14, 178 (1974).

[3] Theory of the Inhomogeneous Electron Gas, Eds. S. Lundqvist, N.H. March, Plenum, New York 1983.

[4] J.A. Bearden, A.F. Burr, Rev. Mod. Phys. 39, 125 (1967).

[5] J. Lindhard, Kgl. Danske Vid. Selsk. Mat.-Fys. Medd. 28, no.8 (1954).

[6] M. Moneta, J. Phys. B At. Mol. Opt. Phys. 32, 1419 (1999).

[7] C.O. Almbladh, U. von Barth, Z.D. Popovic, M. Stott, Phys. Rev. B 14, 2250 (1976).

[8] F.J. Rogers, H.C. Graboske, D.J. Harwood, Phys. Rev. A 1, 1577 (1970). 